\title{
ABUNDANCES IN STARS FROM THE RED GIANT BRANCH TIP TO NEAR THE MAIN-SEQUENCE TURNOFF IN M71. I. SAMPLE SELECTION, OBSERVING STRATEGY, AND STELLAR PARAMETERS ${ }^{1}$
}

\author{
Judith G. COHEN, ${ }^{2}$ BradFord B. Behr, ${ }^{2,3}$ AND Michael M. Briley ${ }^{4}$ \\ Received 2001 March 29; accepted 2001 May 18
}

\begin{abstract}
We present the sample for an abundance analysis of 25 members of $M 71$, with luminosities ranging from the red giant branch tip to the upper main sequence. The spectra are of high dispersion and of high precision. We describe the observing strategy and determine the stellar parameters for the sample stars using both broadband colors and fits of $\mathrm{H} \alpha$ profiles. The derived stellar parameters agree with those from the Yale $^{2}$ stellar evolutionary tracks to within $50-100 \mathrm{~K}$ for a fixed $\log g$, which is within the level of the uncertainties.

Key words: globular clusters: general — globular clusters: individual (M71) — stars: abundances — stars: evolution
\end{abstract}

\section{INTRODUCTION}

By virtue of their large populations of coeval stars, the Galactic globular clusters present us with a unique laboratory for the study of the evolution of low-mass stars. The combination of their extreme ages, compositions, and dynamics also allows us a glimpse at the early history of the Milky Way and the processes operating during its formation. These aspects become even more significant in the context of the star-to-star light element inhomogeneities found among red giants in every globular cluster studied to date. The large differences in the surface abundances of $\mathrm{C}$, $\mathrm{N}, \mathrm{O}$, and often $\mathrm{Na}, \mathrm{Mg}$, and $\mathrm{Al}$ have defied a comprehensive explanation in the three decades since their discovery.

Proposed origins of the inhomogeneities typically break down into two scenarios: (1) $\mathrm{As} \mathrm{C}, \mathrm{N}, \mathrm{O}, \mathrm{Na}, \mathrm{Mg}$, and $\mathrm{Al}$ are related to proton capture processes at $\mathrm{CN}$ - and $\mathrm{CNO}$ burning temperatures, material cycled through a region above the H-burning shell in evolving cluster giants may be brought to the surface with accompanying changes in composition. While standard models of low-mass stars do not predict this "deep mixing," several theoretical mechanisms have been proposed (e.g., the meridional mixing of Sweigart \& Mengel 1979 and turbulent diffusion, Charbonnel 1994, 1995) with varying degrees of success. Moreover, there is ample observational evidence that deep mixing does take place during the red giant branch (RGB) ascent of metalpoor cluster stars (see the reviews by Kraft 1994 and Pinsonneault 1997 and references therein). (2) It has also become apparent that at least some component of these abundance variations must be in place before some cluster stars reach the giant branch. Spectroscopic observations of main-sequence turnoff stars in 47 Tuc (Briley et al. 1996, \& Cannon et al. 1998 and references therein), NGC 6752

\footnotetext{
${ }^{1}$ Based on observations obtained at the W. M. Keck Observatory, which is operated as a scientific partnership among the California Institute of Technology, the University of California, and the National Aeronautics and Space Administration. The Observatory was made possible by the generous financial support of the W. M. Keck Foundation.

2 Palomar Observatory, Mail Stop 105-24, California Institute of Technology, Pasadena, CA 90125.

${ }^{3}$ Current address: Department of Astronomy, University of Texas, Austin, Texas 78712.

${ }^{4}$ Department of Physics, University of Wisconsin, Oshkosh, Oshkosh, Wisconsin.
}

(Suntzeff \& Smith 1991; Gratton et al. 2001) and most recently in M71 (Cohen 1999; Briley \& Cohen 2001) have shown variations in the strength of the $\mathrm{CN}$ and $\mathrm{CH}$ band, and in some cases $\mathrm{Na}$ and $\mathrm{O}$ line strengths as well, consistent with patterns found among the evolved giants of these clusters.

All we know about stellar evolution strongly suggests that these low-mass main-sequence globular cluster stars are incapable of producing significant amounts of $\mathrm{C}, \mathrm{N}$, or $\mathrm{O}$ while on the main sequence and are also incapable of deep dredge-up. Both would be required to reproduce the observed abundance variations. This leads directly to the possibility that the early cluster material was at least partially inhomogeneous in these elements or that some form of modification of the relative abundances of these elements has taken place within the cluster since the currently observed cluster stars were formed. Some suggested culprits include mass loss from intermediate-mass asymptotic giant branch stars and supernovae ejecta (see Cannon et al. 1998 for an excellent discussion of these possibilities).

In addition, King et al. (1998) have added another complication to the issue of abundance variations within globular clusters. Their analysis of a small number of subgiants in M92 yields $[\mathrm{Fe} / \mathrm{H}]=-2.52 \mathrm{dex},{ }^{5}$ a value smaller by about a factor of 2 than that derived from the spectra of giants in M92 by many authors including Cohen (1979) and Sneden et al. (1991). If this is not due to some error in the analysis or a variation in non-LTE corrections that has not been properly included, this result is quite puzzling, since the Fe abundance in the photosphere of these stars could not possibly be altered by mixing.

In an effort to unveil the source of the star-to-star element variations seen among the light elements within globular clusters, as well as to determine the constancy, or lack thereof, of the abundances of the heavy elements such as $\mathrm{Fe}$, where no foreseeable mixing can be expected to alter its abundance, we have in initiated the present program to study at high dispersion stars over a large range in luminosity within the nearer Galactic globular clusters. We begin with the nearest globular cluster easily reached from a northern hemisphere site, M71.

\footnotetext{
${ }^{5}$ The standard nomenclature is adopted; the abundance of element $X$ is given by $[X / \mathrm{H}]=\log [N(X) / N(\mathrm{H})]-\log [N(\mathrm{X}) / N(\mathrm{H})]_{\odot}$.
} 
TABLE 1

The SAMPLe OF Stars IN M71

\begin{tabular}{|c|c|c|c|c|c|c|c|c|}
\hline $\mathrm{ID}^{\mathrm{a}}$ & $\begin{array}{c}V \\
(\mathrm{mag})\end{array}$ & Date Obs. & $\begin{array}{l}\text { Primary } \\
\text { (s) }\end{array}$ & $\begin{array}{l}\mathrm{Al} . \\
(\mathrm{s})\end{array}$ & $\begin{array}{l}\text { Signal pixel } \\
(\mathrm{DN})^{\mathrm{c}}\end{array}$ & $\begin{array}{c}v_{r} \\
\left(\mathrm{~km} \mathrm{~s}^{-1}\right)\end{array}$ & $\begin{array}{c}\mu \text { Prob. }^{\mathrm{b}} \\
(\%)\end{array}$ & Notes \\
\hline $1-45 \ldots \ldots \ldots \ldots \ldots$ & 12.36 & 1999 Aug & 400 & 300 & 2425 & -19.0 & 99 & \\
\hline I $\ldots \ldots \ldots \ldots \ldots \ldots \ldots$ & 12.42 & 1999 Aug & 600 & 400 & 2495 & -13.9 & 99 & \\
\hline $1-66 \ldots \ldots \ldots \ldots \ldots$ & 13.07 & 1999 Aug & 1000 & 400 & 3325 & -23.6 & 96 & \\
\hline $1-64 \ldots \ldots \ldots \ldots \ldots$ & 13.12 & 1999 Aug & 700 & 1200 & 1535 & -17.1 & 99 & \\
\hline $1-56 \ldots \ldots \ldots \ldots \ldots$ & 13.21 & 1999 Aug & 700 & $\ldots$ & 770 & -21.2 & 99 & \\
\hline $1-95 \ldots \ldots \ldots \ldots \ldots$ & 13.35 & 1999 Aug & 1800 & 900 & 3565 & -20.3 & 99 & \\
\hline $1-81 \ldots \ldots \ldots \ldots \ldots$ & 13.68 & 1999 Aug & 1000 & 400 & 1750 & -24.3 & 99 & \\
\hline $\mathrm{Y}, \ldots \ldots \ldots \ldots \ldots \ldots$ & 13.95 & 1999 Aug & 3600 & $\ldots$ & 8800 & -2.1 & 0 & d \\
\hline $1-1 \ldots \ldots \ldots \ldots \ldots \ldots$ & 14.14 & 1999 Aug & 600 & 300 & 675 & -23.5 & 99 & \\
\hline $1-80 \ldots \ldots \ldots \ldots \ldots$ & 14.45 & 2000 June & 900 & $\ldots$ & 4690 & -20.7 & 99 & HB \\
\hline $1-87 \ldots \ldots \ldots \ldots \ldots$ & 14.47 & 2000 June & 2100 & 900 & 2940 & -22.8 & $\ldots$ & HB \\
\hline . $\ldots \ldots \ldots \ldots \ldots$ & 14.58 & 1999 Aug & 1800 & 900 & 975 & -24.6 & 98 & $\mathrm{HB}$ \\
\hline $1-60 \ldots \ldots \ldots \ldots \ldots$ & 14.55 & 1999 Aug & 1800 & $\ldots$ & 1015 & -20.8 & 99 & \\
\hline $1-59 \ldots \ldots \ldots \ldots \ldots$ & 14.71 & 1999 Aug & 1800 & $\ldots$ & 875 & -24.1 & $\ldots$ & \\
\hline G53476_4543..... & 15.07 & 1999 Aug & 7200 & $\ldots$ & 4300 & -22.5 & $\ldots$ & \\
\hline $2-160 \ldots \ldots \ldots \ldots \ldots$ & 15.14 & 2000 June & 2100 & 900 & 1600 & -25.2 & 5 & \\
\hline G53447_4707.................. & 15.16 & 1999 Aug & 7200 & 1200 & 3575 & -19.8 & 28 & \\
\hline G53425_4608........ & 15.47 & 1999 Aug & 1200 & 1200 & 780 & +16.5 & $\begin{array}{r}20 \\
0\end{array}$ & d \\
\hline G53445_4647...... & 15.59 & 2000 June & 3600 & $\ldots$ & 865 & -19.2 & 88 & \\
\hline G53447 $4703 \ldots \ldots$ & 16.03 & 1999 Aug & 7200 & 1200 & 1515 & -27.2 & $\ldots$ & \\
\hline G53425_4612 ....... & 16.32 & 1999 Aug & 1200 & 1200 & 335 & -21.0 & $\begin{array}{l}\cdots \\
\ldots\end{array}$ & \\
\hline G53477 $4539 \ldots \ldots$ & 16.33 & $1999 \mathrm{Aug}$ & 7200 & $\ldots$ & 1255 & -11.4 & $\ldots$ & \\
\hline G53475_4547........ & 16.63 & 1999 Aug & 3600 & $\begin{array}{l}\cdots \\
\cdots\end{array}$ & 600 & +36.3 & $\begin{array}{l}\cdots \\
\cdots\end{array}$ & d \\
\hline G53457_4709...... & 16.75 & 2000 June & 4500 & $\ldots$ & 2960 & -21.1 & $\ldots$ & \\
\hline G53391_4628...... & 16.86 & 1999 Aug & 7200 & $\ldots$ & 1210 & -21.3 & $\ldots$ & \\
\hline G53394_4624..... & 16.95 & 1999 Aug & 4800 & $\begin{array}{l}\cdots \\
\ldots\end{array}$ & 700 & +3.4 & $\begin{array}{l}\cdots \\
\ldots\end{array}$ & d \\
\hline G53417_4431..... & 17.60 & 1999 Aug & 12000 & $\begin{array}{l}\cdots \\
\ldots\end{array}$ & 875 & -19.8 & $\begin{array}{l}\cdots \\
\cdots\end{array}$ & \\
\hline G53392_4624_.... & 17.72 & 1999 Aug & 12000 & $\ldots$ & 820 & -22.1 & $\ldots$ & \\
\hline G53414_4435....... & 17.97 & 1999 Aug & 12000 & $\begin{array}{l}\cdots \\
\cdots\end{array}$ & 425 & -21.4 & $\begin{array}{l}\cdots \\
\cdots\end{array}$ & \\
\hline
\end{tabular}

${ }^{a}$ Identifications are from Arp \& Hartwick (1971) or are assigned based on the J2000 coordinates, rh rm rs.s dd dm dd becoming Grmrss_dmdd.

b This is the probability of membership assigned by Cudworth (1985) on the basis of his proper-motion survey.

${ }^{c}$ The CCD gain is $2.4 e \mathrm{DN}^{-1}$. The signal is measured in the continuum of the spectra taken with the primary HIRES configuration near $6150 \AA$.

d This star is presumed to not be a member of M71.

\section{THE SELECTION OF STARS}

Stars were chosen to span the range from the tip of the red giant branch to the main-sequence turnoff of M71. Membership considerations at this stage involved location

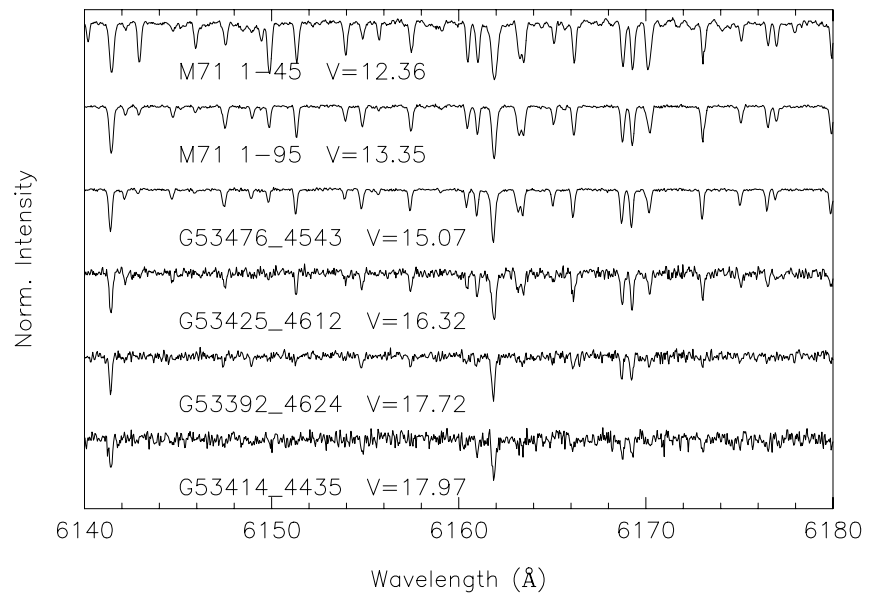

FIG. 1.-Section of order 58 shown for the brightest (at $V$ ) M71 star in our sample at the top of the figure, and the faintest at the bottom. Starting with the brightest star, we display stars in the sample in increments of five in order of decreasing luminosity, omitting the RHB stars. on the existing $B, V$ photometric sequences of Arp \& Hartwick (1971) and, for the more luminous stars, assignment of a high probability of membership by Cudworth (1985) in his proper-motion survey of this globular cluster. When possible, stars were chosen that had known $\mathrm{CH}$ and $\mathrm{CN}$ band strengths from the survey by Briley, Smith, \& Claver (2001) of the red giant branch or the work of Cohen (1999) for the main-sequence region. Unpublished spectra of these bands from Cohen were available for some of the subgiants as well. Within luminosity ranges of $1 \mathrm{mag}$, an effort was made to select stars that spanned the full range of observed $\mathrm{CH}$ and $\mathrm{CN}$ band strengths, i.e., $\mathrm{CN}$ weak and $\mathrm{CN}$ strong stars. Only reasonably isolated stars were selected.

Because this cluster lies at low Galactic latitude, field star contamination is a serious issue. It was not possible to define the cluster sequence clearly in the subgiant regime. There the evolution is rapid, hence the stellar density along the isochrone is low, while the number of field stars is rising rapidly toward fainter magnitudes. Cudworth's (1985) proper-motion survey in M71 does not reach faint enough to include subgiants, and even with the very recent study of Geffert \& Maintz (2000), which was not available at the time our sample was selected, isolation of a clean sample of subgiants in M71 would be quite difficult.

Throughout this paper, the star names are from Arp \& 
Hartwick (1971), or, when not included in the former, are created from the object's J2000 coordinates.

\section{THE HIRES OBSERVATIONS}

All spectra were obtained with HIRES (Vogt et al. 1994) at the Keck Observatory. A maximum slit length of 14" can be used with our instrumental configuration without orders overlapping. Since an image rotator for HIRES is available (built under the leadership of David Tytler), if we can find pairs of program stars with separations less than $8^{\prime \prime}$, they can be observed together on a single exposure. Ideally pairs consisted of two members of the M71 sample, but when that was not feasible, pairs with a random star of suitable brightness (i.e., brighter than the sample star) were chosen in the hope that the second star would also turn out to be a member of M71.

The desired minimum signal-to-noise ratio $(\mathrm{S} / \mathrm{N})$ was 75 over a 4 pixel resolution element for a wavelength near the center of echelle order $56(\sim 6400 \AA)$. This is calculated strictly from the counts in the object spectrum and excludes noise from cosmic-ray hits, sky subtraction, flattening problems, etc. Since the nights were dark, sky subtraction is not an issue except at the specific wavelengths corresponding to strong night-sky emission lines, such as the $\mathrm{Na} \mathrm{D}$ doublet. This $\mathrm{S} / \mathrm{N}$ goal was achieved, at considerable cost in observing time, for all but the faintest star. The faintest star was not a program star, but rather an object that fell within the slit for a program star setup. Its $\mathrm{S} / \mathrm{N}$ is only 50 per resolution element.

The fainter program stars required integration times of several hours. If there was more than one potential brighter second star, then both such could be observed during the course of the exposures for the fainter star by changing the position angle of the instrument's slit at some point during the exposure sequence while still keeping the faint M71 star in the slit.

Approximate measurements of the radial velocity were made on-line, and if a star was determined to be a nonmember, the observations were terminated. If the probable nonmember was the second component in a pair, an attempt was made to switch to another position angle to pick up a different second star, when a possible candidate that was bright enough was available within the limits of the $8^{\prime \prime}$ maximum separation. Through creative use of close pairs, a sample of 29 stars was observed in M71 with HIRES.

To avoid crowding of spectral lines, the observations were centered at about $6500 \AA$. A 1".15 slit was used, which provides a spectral resolution of 34,000. All long integrations were broken up into separate exposures, each $1200 \mathrm{~s}$ long, to optimize cosmic-ray removal.

Because the HIRES detector is undersized, our spectra do not cover the full length of each echelle order without gaps in the wavelength coverage. We wanted to include key lines of critical elements, specifically [O I ] $\lambda \lambda 6300,6363$, the $\mathrm{O}$ $\lambda 7770$ triplet, the $\mathrm{Na} \lambda \lambda 6154,6160$ doublet, and $\mathrm{Al}$ I $\lambda \lambda 6696$, 6698. Two instrumental configurations were used for the brightest stars, as it was impossible to create a single one which included all the desired spectral features in the wavelength range 6000 to $8000 \AA$. In particular, although the Al I $\lambda \lambda 6696,6698$ doublet is the most useful feature of that element in this spectral region, we could not get it to fit into a single instrumental configuration together with the $\mathrm{O}$ lines. For the faintest stars, only a single configuration was used, which included the $\mathrm{O}$ lines but did not include the Al I doublet.
The spectra were reduced by B. B. B. using FIGARO (Shortridge 1993) scripts with commands written by McCarthy and Tomaney (McCarthy 1988) specifically for echelle data reduction.

Table 1 gives details of the HIRES exposures for each star, with the total exposure time for the primary and for the Al configuration. The signal level per pixel in the continuum at $6150 \AA$ is also given, from which the $\mathrm{S} / \mathrm{N}$ can be calculated assuming Poisson statistics and ignoring issues of cosmic-ray removal, flattening, etc. The latter become nonnegligible for the very long HIRES integrations necessary as faint as possible in M71. Also listed is the radial velocity for each star measured from the HIRES spectra and the probability of membership assigned by Cudworth (1985) based on his proper-motion study, which included only the brighter stars in the sample. A montage of spectra of a single echelle order for selected stars covering the luminosity range of the sample is shown in Figure 1.

It should be noted that to acquire this set of relatively high-precision and high-dispersion spectra took a total of five nights of assigned time at the Keck Observatory. One of the assigned nights was used for a backup program because of poor seeing conditions.

\section{RADIAL VELOCITIES}

Radial velocities were measured from all the HIRES spectra by cross-correlating orders 56 and 57 against the spectrum of a bright template star (in practice the brightest observed M71 star) from each run. The radial velocity of the template star from each run was determined by fitting Gaussians to 20 strong isolated features in these two orders using wavelengths from the NIST Atomic Spectra Database Version 2.0 (NIST Standard Reference Database No. 78). Heliocentric corrections appropriate for each exposure were then applied.

The internal radial velocity errors were calculated following the precepts of Tonry \& Davis (1979) using the relation $\sigma\left(v_{\mathrm{r}}\right)=\alpha /[1+R(\mathrm{TD})]$, where the parameter $R(\mathrm{TD})$ is a measure of the ratio of the height of the peak of the crosscorrelation to the noise in the cross-correlation function away from the peak. The constant $\alpha$ was set at $15 \mathrm{~km} \mathrm{~s}^{-1}$, which represents a value at the upper end of those found in other recent HIRES programs using similar instrumental configurations by Mateo et al. (1998), Cook et al. (1999), and Côté et al. (1999). Every star except the faintest one observed, G53414_4435, has an internal error in $v_{r}$ under $1 \mathrm{~km} \mathrm{~s}^{-1}$, while this star only has a somewhat larger internal error of $1.5 \mathrm{~km} \mathrm{~s}^{-1}$.

Based on their measured radial velocities, four of the 29 stars observed with HIRES are not members of M71 (stars Y, G53425_4608, G53475_4547, and G53394_4624). All of these four stars were chosen not as members of the primary M71 sample but as bright(er) stars to complete a pair, i.e., chosen primarily on the basis of their location on the sky without as careful scrutiny of their colors as was done for the primary sample.

The radial velocities for the 25 members of M71 observed with HIRES are listed in Table 1 . They have a mean $v_{r}$ of $-21.7 \mathrm{~km} \mathrm{~s}^{-1}$. After removing in quadrature an internal uncertainty of $1.0 \mathrm{~km} \mathrm{~s}^{-1}$, we find $\sigma=2.6 \mathrm{~km} \mathrm{~s}^{-1}$. This is in excellent agreement with the value of Peterson \& Latham (1986) of $-22.5 \mathrm{~km} \mathrm{~s}^{-1}\left(\sigma=2.4 \mathrm{~km} \mathrm{~s}^{-1}\right)$ determined from a sample of 17 bright giants in M71.

The only star that shows lines obviously broader than 
those expected from the instrumental resolution is M71 I-80, a RHB star. The profiles of weak lines of this star suggest that it is rotating at a projected velocity of $\sim 8 \mathrm{~km}$ $\mathrm{s}^{-1}$, an issue that will be discussed by $\mathrm{B}$. Behr in a future publication.

\section{THE STELLAR PARAMETERS}

We seek to determine for the M71 sample of stars the stellar atmosphere parameters necessary to carry out an abundance analysis from our HIRES data. We adopt for M71 the distance $(3900 \mathrm{pc})$ and reddening $[E(B-V)=0.25$ mag] from the on-line compilation of Harris (1996). ${ }^{6}$ The relative extinction in various passbands is taken from Cohen et al. 1981; see also Schlegel, Finkbeiner, \& Davis 1998). Based on the high-dispersion analysis of Cohen (1983) for several red giants in M71, since confirmed by additional high-resolution studies of giants in M71 by Leep, Oke, \& Wallerstein (1987) and by Sneden et al. (1994), we adopt as an initial guess a metallicity for the cluster of $[\mathrm{Fe} / \mathrm{H}]=-0.7$ dex.

${ }^{6}$ The extinction maps of Schlegel et al. (1998) from their analysis of the $C O B E / D I R B E$ database are not reliable this close to the Galactic plane. For M71, with $b=-4.6$, they deduce $E(B-V)=0.32 \mathrm{mag}$.

\section{1. $T_{\text {eff }}$ from Broadband Colors: Predictions of the Model Atmospheres}

We utilize here the grid of predicted broadband colors and bolometric corrections of Houdashelt, Bell, \& Sweigart (2000) based on the MARCS stellar atmosphere code (Gustafsson et al. 1975). Before proceeding, we demonstrate that the Kurucz and MARCS predicted colors are essentially identical, at least for the specific colors used here.

We compare the colors predicted from the MARCS code from Houdshelt et al. with those from the Kurucz ATLAS code (Kurucz 1992). We take the predicted $V-K$ color from each model in the MARCS grid with $[\mathrm{Fe} / \mathrm{H}]=-0.5$, and interpolate within the Kurucz color grid at the same abundance and at the $\log g$ of the MARCS model to find the $T_{\text {eff }}$ that would be deduced.

A contour plot of the difference $\Delta T_{\text {eff }}$ (Kurucz-MARCS) that results when the $V-K$ color is used is shown in Figure 2. The three contour levels shown correspond to $\Delta T_{\text {eff }}=0$, 30 , and $60 \mathrm{~K}$. Also shown in this figure as the thick curve is a $12 \mathrm{Gyr}$ isochrone for M71 from the very recently completed $\mathrm{Yale}^{2}$ isochrones of $\mathrm{Yi}$ et al. (2001). Along this isochrone, $\Delta T_{\text {eff }}=0$ to $25 \mathrm{~K}$ for the subgiants and main-sequence turnoff, and $\Delta T_{\text {eff }}=0$ to $15 \mathrm{~K}$ for the red giant branch. Throughout the entire displayed range in $T_{\text {eff }}$,

TABLE 2

PHOTOMETRY FOR THE M71 SAMPLE

\begin{tabular}{|c|c|c|c|c|c|c|}
\hline $\mathrm{ID}^{\mathrm{a}}$ & $\begin{array}{c}V \\
(\mathrm{mag})\end{array}$ & $\begin{array}{c}R \\
(\mathrm{mag})\end{array}$ & $\begin{array}{c}I \\
(\mathrm{mag})\end{array}$ & $\begin{array}{c}K \\
(\mathrm{mag})\end{array}$ & R.A. & Decl. \\
\hline \multicolumn{7}{|l|}{ Probable members: } \\
\hline 1-45 .............. & 12.36 & $\ldots$ & $\ldots$ & 8.12 & 195348.37 & +184800.3 \\
\hline I $\ldots \ldots \ldots \ldots \ldots \ldots$ & 12.423 & $\ldots$ & 10.76 & 8.56 & 195344.74 & +184635.1 \\
\hline $1-66 \ldots \ldots \ldots \ldots \ldots$ & 13.071 & $\ldots$ & 11.49 & $\ldots$ & 195345.22 & +184655.5 \\
\hline $1-64 \ldots \ldots \ldots \ldots \ldots$ & 13.122 & $\ldots$ & 11.49 & 9.32 & 195346.12 & +184726.2 \\
\hline $1-56 \ldots \ldots \ldots \ldots \ldots$ & 13.21 & $\ldots$ & 11.77 & 9.79 & 195348.40 & +184823.5 \\
\hline $1-95 \ldots \ldots \ldots \ldots \ldots$ & 13.35 & $\ldots$ & 11.94 & $\ldots$ & 195341.01 & +184604.8 \\
\hline $1-81 \ldots \ldots \ldots \ldots \ldots$ & 13.68 & $\ldots$ & 12.184 & 10.22 & 195345.48 & +184649.7 \\
\hline $1-1 \ldots \ldots \ldots \ldots \ldots$ & 14.14 & $\ldots$ & 12.76 & 10.92 & 195352.32 & +184452.9 \\
\hline $1-80 \ldots \ldots \ldots \ldots \ldots$ & 14.45 & 13.85 & 13.29 & $\ldots$ & 195344.20 & +184648.0 \\
\hline $1-87 \ldots \ldots \ldots \ldots \ldots$ & 14.47 & 13.83 & 13.28 & $\ldots$ & 195345.58 & +184548.9 \\
\hline $1-94 \ldots \ldots \ldots \ldots \ldots$ & 14.58 & 13.94 & 13.42 & 12.01 & 195340.85 & +184600.9 \\
\hline $1-60 \ldots \ldots \ldots \ldots \ldots$ & 14.55 & 13.83 & 13.23 & 11.69 & 195341.78 & +184841.5 \\
\hline $1-59 \ldots \ldots \ldots \ldots \ldots$ & 14.71 & 13.92 & 13.22 & 11.39 & 195342.06 & +184837.3 \\
\hline G53476_4543...... & 15.07 & 14.38 & 13.77 & $\ldots$ & 195347.62 & +184543.2 \\
\hline $2-160 \ldots \ldots \ldots \ldots$ & 15.14 & 14.46 & 13.89 & $\ldots$ & 195345.19 & +184833.2 \\
\hline G53447_4707...... & 15.16 & 14.48 & 13.94 & 12.50 & 195344.65 & +184707.4 \\
\hline G53445_4647...... & 15.59 & 14.88 & $14.31^{\mathrm{b}}$ & $\ldots$ & 195344.50 & +184647.0 \\
\hline G53447_4703...... & 16.03 & 15.27 & 14.74 & 13.20 & 195344.65 & +184703.3 \\
\hline G53425_4612..... & 16.32 & 15.66 & 15.13 & $\ldots$ & 195342.45 & +184611.7 \\
\hline G53477_4539..... & 16.33 & 15.66 & 15.10 & $\ldots$ & 195347.72 & +184539.2 \\
\hline G53457_4709...... & 16.75 & 16.02 & 15.57 & $\ldots$ & 195345.69 & +184708.8 \\
\hline G53391_4628...... & 16.86 & 16.19 & 15.60 & $\ldots$ & 195339.05 & +184628.2 \\
\hline G53417_4431..... & 17.60 & 17.05 & 16.52 & 15.49 & 195341.72 & +184431.2 \\
\hline G53392_4624 ...... & 17.72 & 17.13 & 16.66 & $\ldots$ & 195339.18 & +184623.9 \\
\hline G53414_4435..... & 17.97 & 17.44 & $\ldots$ & 15.78 & 195341.37 & +184434.8 \\
\hline \multicolumn{7}{|l|}{ Probable nonmembers: } \\
\hline Y . .................. & 13.95 & $\ldots$ & 12.62 & 11.13 & 195341.31 & +184427.4 \\
\hline G53425_4608...... & 15.47 & 14.68 & 14.08 & $\ldots$ & 195342.48 & +184607.7 \\
\hline G53475_4547......... & 16.63 & 16.13 & 15.80 & $\cdots$ & 195347.53 & +184547.3 \\
\hline G53394_4624 ...... & 16.95 & 16.35 & 15.91 & $\ldots$ & 195339.41 & +184623.8 \\
\hline
\end{tabular}

NotE.-Units of right ascension are hours, minutes, and seconds, and units of declination are degrees, arcminutes, and arcseconds (J2000.0).

${ }^{a}$ Identifications are from Arp \& Hartwick (1971) or are assigned based on the J2000 coordinates, rh rm rs.s dd dm dd becoming Grmrss_dmdd.

b This star has $V, I$ from Stetson, as well as from the short LRIS images, but Stetson's $I$ is very discrepant from that from the LRIS short images. This is the only such case found so far. 


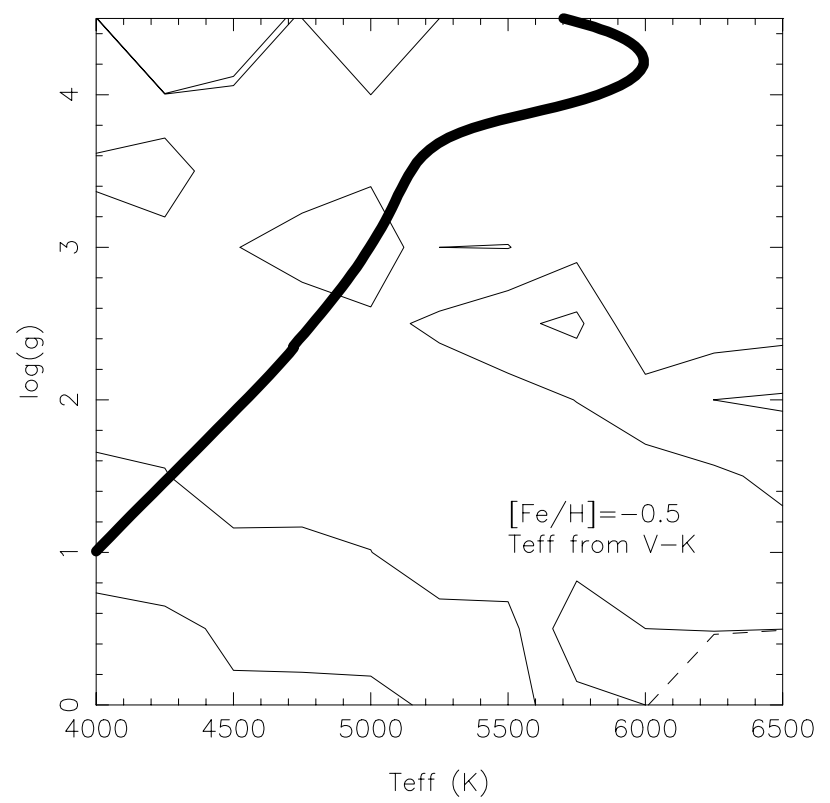

Fig. 2.-Contours of $\Delta T_{\text {eff }}$ computed for $V-K$ displayed to demonstrate that the Kurucz and MARCS grids of predicted colors are essentially identical. $\Delta T_{\text {eff }}$ is the difference between the $T_{\text {eff }}$ predicted from the ATLAS models of Kurucz (1992) and the MARCS models of Houdshelt et al. (2000) for a fixed abundance $([\mathrm{Fe} / \mathrm{H}]=-0.5 \mathrm{dex})$ and a $V-K$ color taken from the MARCS grid. The contour levels are 0,30 , and $60 \mathrm{~K}$. The thick curve is a 12 Gyr isochrone for M71 taken from the Yale ${ }^{2}$ tracks of Yi et al. (2001). See the text for details.

$\log g$ ), $\delta T_{\text {eff }}$ ranges from 0 to $50 \mathrm{~K}$. We thus demonstrate that to within a tolerance of $25 \mathrm{~K}$, the Kurucz and MARCS temperature scales from broadband $V-K$ colors are identical.

\section{2. $T_{\text {eff }}$ from Broadband Colors}

Broadband $B, V$ colors are available from the work of Arp \& Hartwick (1971). With such high reddening and metallicity, we chose to ignore the $B$ measurements. Stetson (2000) provides $V, I$ photometry for about $25 \%$ of the cluster area, specifically the northeast quadrant. To supplement this, J. G. C. carried out $V, R, I$ photometry using short exposure images of M71 taken with LRIS (Oke et al. 1995) at the Keck Observatory for slit-mask alignment purposes. These frames were calibrated by observations of standard fields from Landolt (1992). The brightest M71 giants were saturated on all the LRIS images.

For a smaller sample of $\sim 235$ stars in M71, we have obtained infrared photometry at $K$ using the infrared acquisition and guiding camera on NIRSPEC (McLean et al. 1998, 2000) at the Keck Observatory. These were taken during a night dedicated to infrared spectroscopy in M71, and are basically setup images for the spectroscopy. The data are calibrated to the standard stars of Persson et al. (1998). A $256 \times 256$ pixel NICMOS detector is used with a scale of $0^{\prime \prime} 18$ pixel $^{-1}$. Hence the fields are very small and main-sequence stars dominate the sample. The frames were reduced in a standard manner. This is supplemented by the infrared photometry of Frogel, Persson, \& Cohen (1979) for the upper giant branch of M71. For the single star in common between the two infrared samples, there is reasonable agreement (see below). Infrared photometry was obtained for a few additional stars using the camera of Murphy et al. (1995) at the $1.5 \mathrm{~m}$ telescope at Palomar Mountain. With a scale of 0.6 pixel $^{-1}$ and a $256 \times 256$
NICMOS array, only a small portion of M71 can be covered at once. Exposures of more than $2000 \mathrm{~s}$ (broken up into many spatial positions and repeats) are required to reach the fainter stars in our sample, and the crowding is severe with these relatively large pixels. However, $K$ magnitudes for five of the stars in the HIRES sample were obtained in this way.

The $V, V-K$ color magnitude diagram for M71 for the HIRES sample is shown in Figure 3. The different sources of $K$ photometry are indicated by different symbols. The stars observed by Frogel et al. (1979) are also indicated; three stars from their sample, whose membership probabilities are below $15 \%$ in the proper-motion study of Cudworth (1985), have been excluded as it is unlikely that they are members of M71. There are two stars in the HIRES sample with multiple measurements for $K$. These are indicated by horizontal lines connecting the relevant points in this figure. (M71 1-45, the brightest star in $V$ in the HIRES sample, near the tip of the RGB has two measurements of $K$ differing by only $0.09 \mathrm{mag}$; difficult to see on the figure.)

The photometry of Frogel et al. (1979) reaches to RHB. While the number of stars in common with the previously published photometry is only one, the consistency of the M71 RGB and RHB delineated by the published photometry and by our photometry shown in Figure 3 indicates that our mixing of several different sources for $K$ has been done in a valid manner.

The observed broadband colors for each program star are given in Table $2 V-I$ and, when available, $V-K$, corrected for extinction, are used to determine $T_{\text {eff }}$. The set of

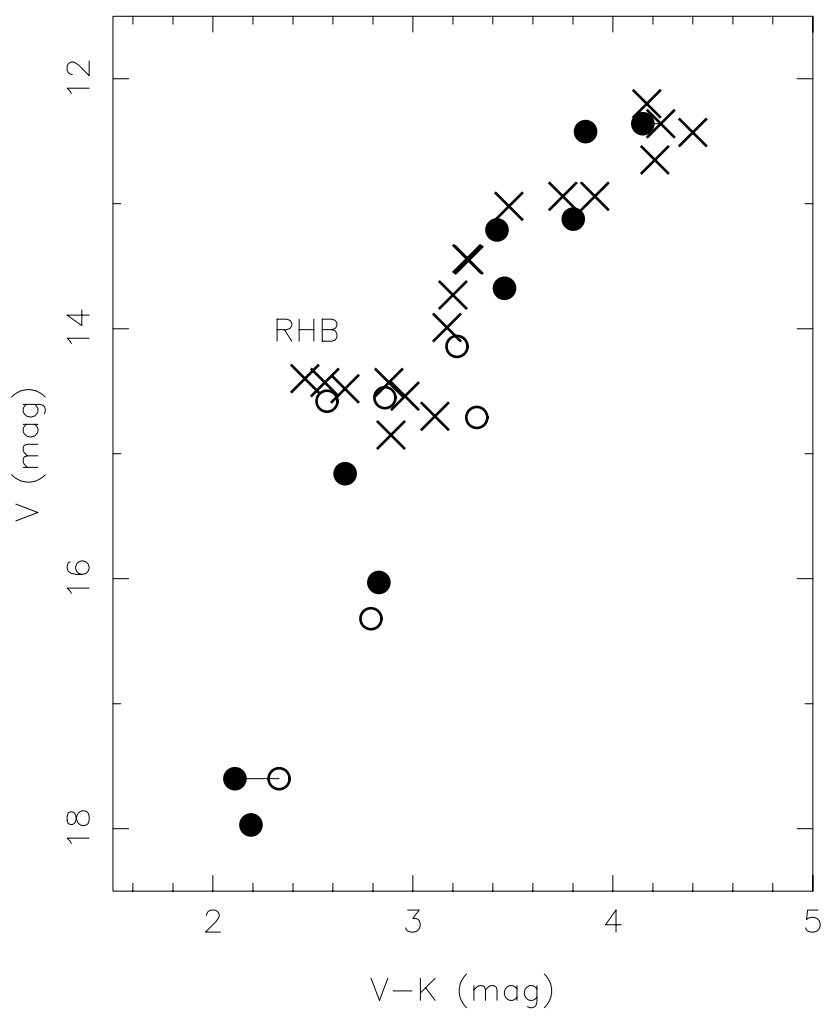

FIG. 3. $-V, V-K$ color-magnitude diagram for M71 for the HIRES sample. Filled circles denote measurements from SCAM/NIRSPEC, open circles denote measurements from the P60 IR camera, and crosses denote the set of RGB and RHB stars from Frogel et al. (1979). For each of the two stars with more than one independent observation at $K$, horizontal lines connect the pair of points. 
models with metallicity of -0.5 dex, nearest to our initial estimate of $[\mathrm{Fe} / \mathrm{H}]$, is used. Table 3 lists the $T_{\text {eff }}$ deduced from each of $V-I$ and $V-K$, when available.

The calibration of our photometric data, as distinct from that of Stetson (2000), could be better. We assume an uncertainty of 0.02 mag applies to $V-I$ from Stetson (2000), an uncertainty of $0.03 \mathrm{mag}$ applies to colors from the LRIS images, and an uncertainty of $0.05 \mathrm{mag}$ applies to all $V-K$ colors. Given the relatively high reddening of M71, there is an additional uncertainty due to possible spatial variations in reddening across the field of the cluster. We assume this occurs for $E(B-V)$ at a level of $10 \%$, which is the fractional variation in $E(B-V)$ detected across much more heavily reddened globular clusters by Cohen \& Sleeper (1995). This translates into a total uncertainty in $T_{\text {eff }}$ of $75 \mathrm{~K}$ for giants rising to $150 \mathrm{~K}$ for main-sequence stars using $V-I$, divided about equally between the two contributions (uncertainty in reddening and photometric colors), and $40 \mathrm{~K}$ from $V-K$ for giants rising to $70 \mathrm{~K}$ for dwarfs, with the dominant contribution arising from the photometric uncertainties. We adopt the larger of these uncertainties (those from $V-I$ ) as applicable for our $T_{\text {eff }}$ determinations.

\subsection{Computation of $\log g$}

Once an initial guess at $T_{\text {eff }}$ has been established from a broadband color, it is possible with minimal assumptions to evaluate $\log g$ using observational data. The adopted distance modulus, initial guess at $T_{\text {eff }}$, and an assumed stellar mass (we adopt $0.8 M_{\odot}$ except for the RHB stars for which we adopt $0.6 M_{\odot}$ ) are combined with the known interstellar absorption, the predictions of the model atmosphere grid for bolometric corrections, as well as a broadband observed $V$ mag, to calculate $\log g$.

An iterative scheme is used to correct for the small dependence of the predictions of the model atmosphere grid on $\log g$ itself. Rapid convergence is achieved.

It is important to note that because of the constraint of a known distance to M71, the uncertainty in $\log g$ is small, $\leq 0.1 \mathrm{dex}$, when comparing two members of M71. Propagating an uncertainty of $15 \%$ in the cluster distance, $5 \%$ in the stellar mass, and $3 \%$ in $T_{\text {eff }}$ from a reddening uncertainty of $0.04 \mathrm{mag}$ in $E(B-V)$ and ignoring any covariance leads to a potential systematic error of $\pm 0.2 \mathrm{dex}$ for $\log g$.

\section{4. $T_{\text {eff }}$ and $\log g$ from $\mathrm{H} \alpha$ Profiles}

The profiles of the Balmer lines can also be used in principle to determine $T_{\text {eff }}$ and $\log g$ in the temperature range characteristic of the M71 stars. The estimates of stellar parameters obtained in this way are to first order independent of reddening and of any photometric data. There is little sensitivity to surface gravity or overall abundance. Given the constraints on $\log g$ and known approximate metallicity imposed by the globular cluster membership of the sample stars, the primary dependence of the Balmer line profiles in this regime of $T_{\text {eff }}$ and $\log g$ for the HIRES sample of members of M71 is on $T_{\text {eff }}$.

We attempt to use the $\mathrm{H} \alpha$ profiles for this purpose. The HIRES spectra themselves are not suitable for this purpose,

TABLE 3

Stellar Parameters for the M71 Sample

\begin{tabular}{|c|c|c|c|c|c|c|}
\hline \multirow[b]{2}{*}{$\mathrm{ID}^{\mathrm{a}}$} & \multicolumn{2}{|c|}{$T_{\text {eff }}(\mathrm{K})$} & \multirow[b]{2}{*}{$\log g$} & \multicolumn{3}{|c|}{$T_{\text {eff }}(\mathrm{K})$} \\
\hline & $(V-K)$ & $(V-I)$ & & $\mathrm{H} \alpha$ & Weighted $^{\mathrm{b}}$ & Adopted \\
\hline $1-45 \ldots \ldots \ldots \ldots \ldots$ & 3950 & & 0.9 & $\ldots$ & 3950 & 3950 \\
\hline I $\ldots \ldots \ldots \ldots \ldots \ldots$ & 4120 & 4175 & 1.0 & $\ldots$ & 4150 & 4150 \\
\hline $1-66 \ldots \ldots \ldots \ldots \ldots$ & $\ldots$ & 4305 & 1.35 & $\ldots$ & 4310 & 4250 \\
\hline $1-64 \ldots \ldots \ldots \ldots \ldots$ & 4170 & 4225 & 1.35 & $\ldots$ & 4200 & 4200 \\
\hline $1-56 \ldots \ldots \ldots \ldots \ldots$ & 4475 & 4560 & 1.6 & $\ldots$ & 4525 & 4525 \\
\hline $1-95 \ldots \ldots \ldots \ldots \ldots$ & $\ldots$ & 4630 & 1.65 & $\ldots$ & 4630 & 4550 \\
\hline $1-81 \ldots \ldots \ldots \ldots \ldots$ & 4450 & 4460 & 1.75 & $\ldots$ & 4455 & 4550 \\
\hline $1-1 \ldots \ldots \ldots \ldots \ldots$ & 4625 & 4710 & 2.05 & $\ldots$ & 4670 & 4700 \\
\hline $1-80^{c} \ldots \ldots \ldots \ldots \ldots$ & $\ldots$ & 5290 & 2.45 & $\ldots$ & 5290 & 5300 \\
\hline $1-87^{c} \ldots \ldots \ldots \ldots \ldots$ & $\ldots$ & 5205 & 2.45 & $\ldots$ & 5205 & 5300 \\
\hline $1-94^{c} \ldots \ldots \ldots \ldots \ldots$ & 5320 & 5290 & 2.45 & $\ldots$ & 5315 & 5300 \\
\hline $1-60 \ldots \ldots \ldots \ldots \ldots$ & 4980 & 4845 & 2.3 & $\ldots$ & 4910 & 4900 \\
\hline $1-59 \ldots \ldots \ldots \ldots \ldots$ & 4560 & 4480 & 2.3 & $\ldots$ & 4520 & 4600 \\
\hline G53476_4543..... & $\ldots$ & 4890 & 2.65 & $\ldots$ & 4890 & 4900 \\
\hline $2-160 \ldots \ldots \ldots \ldots \ldots$ & $\ldots$ & 5175 & 2.7 & $\ldots$ & 5175 & 5100 \\
\hline G53447_4707...... & 5225 & 5130 & 2.75 & 5300 & 5200 & 5175 \\
\hline G53445_4647..... & $\ldots$ & 4960 & 2.85 & $\ldots$ & 4960 & 5050 \\
\hline G53447_4703...... & 5035 & 4920 & 3.0 & $\ldots$ & 4985 & 5000 \\
\hline G53425_4612..... & 5090 & 5205 & 3.15 & 5100 & 5140 & 5150 \\
\hline G53477_4539..... & $\ldots$ & 5090 & 3.15 & 5300 & 5160 & 5150 \\
\hline G53457_4709..... & $\ldots$ & 5240 & 3.35 & 5400 & 5290 & 5200 \\
\hline G53391_4628...... & $\ldots$ & 5010 & 3.35 & $\ldots$ & 5010 & 5100 \\
\hline G53417_4431..... & 6010 & 5580 & 4.05 & $5800^{d}$ & 5800 & 5800 \\
\hline G53392_4624...... & $\ldots$ & 5650 & 4.05 & $5800^{d}$ & 5650 & 5800 \\
\hline G53414_4435..... & 5895 & $\ldots$ & 4.15 & $5800^{d}$ & 5895 & 5900 \\
\hline
\end{tabular}

${ }^{\text {a }}$ Identifications are from Arp \& Hartwick (1971) or are assigned based on the J2000 coordinates, rh rm rs.s dd dm dd becoming Grmrss_dmdd.

${ }^{b} T_{\text {eff }}$ from the $\mathrm{H} \alpha$ profile has half weight.

c These are RHB stars in M71.

d $\mathrm{The} \mathrm{H} \alpha$ profiles are not used to derive $T_{\text {eff }}$ for these stars. 
as the large scale continuum determination, particularly in these relatively metal rich cool stars, is suspect at the level of $1 \%$ to $2 \%$ because of the imperfect removal of the variation of the instrumental response across each echelle order. Instead the Balmer line profiles were obtained from observations with LRIS (Oke et al. 1995) at the Keck Observatory. A 1200 groove $\mathrm{mm}^{-1}$ grating with 0 ".7 wide slits was centered at $6500 \AA$ to yield $0.63 \AA$ pixel $^{-1}$ or a spectral resolution of $\sim 1.7 \AA$. The same slit masks that were designed, fabricated, and utilized for the $\mathrm{CH}$ and $\mathrm{CN}$ observations of 79 main-sequence stars in M71 described in Cohen (1999) were used again in M71 for these observations. An additional slit mask of subgiants was also designed and used for this purpose.

The spectra were reduced in the usual fashion using FIGARO (Shortridge 1993). Continuum bandpasses were defined based on examination of the much higher dispersion HIRES spectra of M71 stars. The median value within each of the continuum regions was chosen as a representative value for the bandpass. A second-order polynomial fit to the signal for each of the "line-free" regions was used to define the continuum. To improve the $\mathrm{S} / \mathrm{N}$ still further for the main-sequence stars, the $\mathrm{H} \alpha$ spectra of three to five stars of similar luminosity along the main sequence of M71 were summed, then the resulting profile was normalized. Figure 4 shows $\mathrm{H} \alpha$ profiles for three stars summed near the bright end of Cohen's (1999) main-sequence sample, which corresponds in the color-magnitude diagram of M71 to the main-sequence stars in the HIRES sample, as well as for a subgiant.

These profiles were compared against the spectral flux calculated by Hauschildt et al. (1999) for $[\mathrm{Fe} / \mathrm{H}]=-0.7$ dex. ${ }^{7}$ The grid spacing of the spectral synthesis is $2 \AA$. We fit the predicted flux of Hauschildt et al. in the region of $\mathrm{H} \alpha$ using the same procedure as had been applied to the stellar spectra to generate a set of model Balmer line profiles with a normalized continuum level.

${ }^{7}$ As has already been pointed out by van't Veer-Menneret \& Megessier (1996), the predicted Balmer line profiles released with the ATLAS9 models of Kurucz (1992) are not valid and fail to reproduce the solar profile. They found, as do we, that these $\mathrm{H} \alpha$ profiles overestimate $T_{\text {eff }}$ by several hundred degrees.

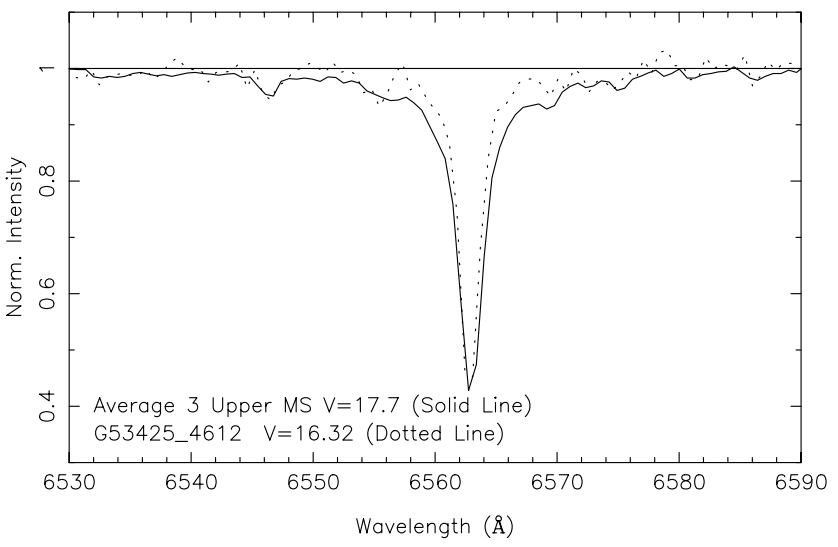

FIG. 4.-Profiles of $\mathrm{H} \alpha$ from LRIS spectra are shown for a subgiant and for the average of three main-sequence stars near the bright end of the sample of Cohen (1999).
Even with the use of LRIS spectra instead of echelle spectra, the continuum determination across the $\mathrm{H} \alpha$ profile is still uncertain by $1 \%$. The sensitivity to $T_{\text {eff }}$ of the wings of the predicted $\mathrm{H} \alpha$ profiles is not large compared with this potential uncertainty. To minimize its effect, we compared the observed and predicted $\mathrm{H} \alpha$ profiles only over the region within $3 \AA$ of the line center. The resulting values of $T_{\text {eff }}$ for our M71 stars are still considerably higher than those derived from the broadband colors. Because of the problems mentioned above, as well as the potential impact of continued small improvements to the broadening theory for Balmer lines (see, for example, Barklem, Piskunov, \& O'Mara 2000), we decided to use the $\mathrm{H} \alpha$ profiles only to estimate relative values for $T_{\text {eff }}$ from star to star within the M71 sample, forcing agreement with the $T_{\text {eff }}$ deduced from the colors at the main sequence.

The $\mathrm{H} \alpha$ profiles provide measurements of $T_{\text {eff }}$ which are in agreement with those derived from the stellar colors to within the uncertainties of each method. One might hope to determine the stellar mass at the turnoff directly from the observations through the gravity dependence of these Balmer line profiles. However, the required precision in the observed Balmer line profiles of better than $1 \%$ is not easily achieved, nor is it clear that the theoretical profiles are sufficiently accurate. Furthermore, the dependence of the Balmer line profiles on $T_{\text {eff }}$ is much larger. Thus determining $T_{\text {eff }}$ itself with sufficient precision to then extract a precise value for $\log g$ would be extremely difficult.

\section{COMPARISON OF STELLAR PARAMETERS WITH ISOCHRONES}

Table 3 provides a summary of the stellar parameters for the 25 members of our M71 sample determined both from broadband photometry and from $\mathrm{H} \alpha$ fits. In addition to the values of $T_{\text {eff }}$ from $V-I$, from $V-K$, and from $\mathrm{H} \alpha$ (when appropriate), ${ }^{8}$, a mean temperature is listed. The weight of the $\mathrm{H} \alpha$ value, when used, is half that of the values from $V-I$ and from $V-K$. With the adopted zero point for assignment of $T_{\text {eff }}$ from the $\mathrm{H} \alpha$ profiles, the good agreement between the three values, consistent with the expected observational errors, is gratifying.

Given that these stars sample the population of a globular cluster, $T_{\text {eff }}$ should decrease monotonically as the luminosity of the star increases. Furthermore, stars in the same region of the cluster isochrone ideally should have very similar stellar parameters. The weighted values of $T_{\text {eff }}$ given in Table 3 do not quite achieve this. We therefore slightly adjusted the weighted $T_{\text {eff }}$, by not more than $100 \mathrm{~K}(150 \mathrm{~K}$ for star G53392_4624) (values typical of our observational uncertainties), while retaining the mean relationship unaltered to try to achieve this. The adopted $T_{\text {eff }}$ for each star in the M71 sample, listed in the final column of Table 3, is the value used in the abundance analyses presented in subsequent papers in this series.

For the nonmembers, since their distances are unknown, no value of $\log g$ can be obtained and the derived $T_{\text {eff }}$ will be incorrect if the reddening is different from the value adopted for M71.

Figure 5 compares the adopted $T_{\text {eff }}$ and $\log g$ for our HIRES sample of members of M71 with the isochrone pre-

\footnotetext{
${ }^{8} \mathrm{H} \alpha$ is not included in the determination of $T_{\text {eff }}$ for the three hottest stars near the M71 main-sequence turnoff.
} 


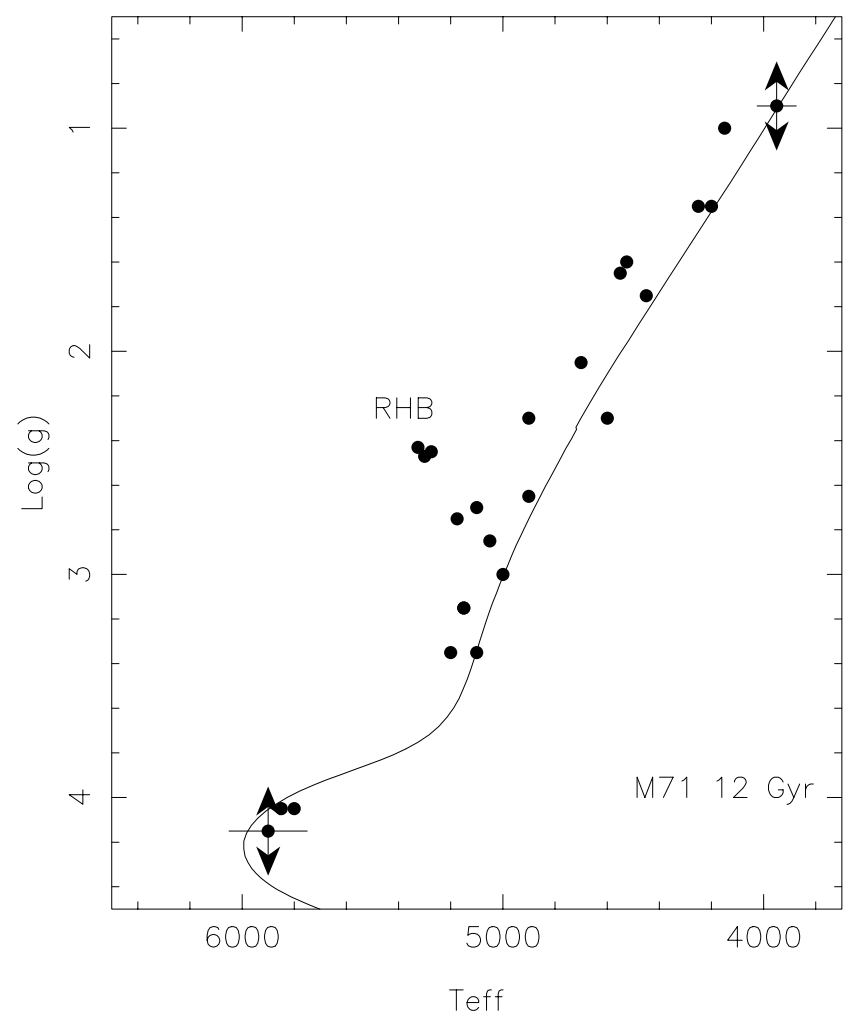

FIG. 5.- $T_{\text {eff }}$ and $\log g$ deduced here for the sample of M71 members with HIRES spectra, as well as the $12 \mathrm{Gyr}^{\mathrm{Yale}^{2}}$ isochrone of $\mathrm{Yi}$ et al. (2001) for $[\mathrm{Fe} / \mathrm{H}]=-0.7 \mathrm{dex}$ (solid curve). A distance of $3900 \mathrm{pc}$ with a reddening $E(B-V)=0.25 \mathrm{mag}$ has been adopted. The arrows in $\log g$ indicate the systematic error which is dominated by the contribution from the distance uncertainty; the internal error from star to star within M71 is considerably smaller. The error bars in $T_{\text {eff }}$ shown for the most and least luminous M71 stars in the HIRES sample are dominated by uncertainties in the reddening and are typical of the sample.

dicted for a stellar system with an age of $12 \mathrm{Gyr}$ with [Fe/ $\mathrm{H}]=-0.7$ dex from the very recently completed Yale $^{2}$ tracks of Yi et al. (2001). Scaled solar mixture abundances are used in the Yale $^{2}$ calculations for all elements heavier than $\mathrm{He}$.

First we note that the set of M71 stars observed with HIRES provides a reasonable sample of the cluster isochrone from the RGB tip to the upper main sequence, with the exception of the lack of subgiants.

Comparing theory and observation using the set of parameters shown in Figure 5, quite different from the usual color-magnitude diagram, is a very stringent test. The agreement with the new $\mathrm{Yale}^{2}$ isochrone is quite good. The $T_{\text {eff }}$ of the theoretical giant branch for the metallicity of M71, which is well known from past work and determined yet again in Ramírez et al. (2001, hereafter Paper II) is only $50-100 \mathrm{~K}$ cooler at a fixed $\log g$ than are the observed stars.

We already know from comparison with the infrared photometry of Frogel et al. (1979) that one cannot ascribe this systematic discrepancy to uncertainties in the measurements. So we now consider the various types of systematic errors that might have occurred. There are two known systematic errors in the handling of the observational data described above. The first is a systematic underestimate of $\log g$ by 0.04 dex as the theoretically predicted mass along the upper RGB is $0.88 M_{\odot}$, while a mass of $0.80 M_{\odot}$ was used to calculate the surface gravities for the cluster stars (excluding the RHB stars) from the observed magnitudes and colors. The second is an underestimate in $T_{\text {eff }}$ of less than $20 \mathrm{~K}$ because the model grid used to define the predicted broadband colors had $[\mathrm{Fe} / \mathrm{H}]=-0.5 \mathrm{dex}$, not the nominal metallicity of M71 of -0.7 dex.

As discussed in $\S 5.3$, the internal errors from star to star in $\log g$ are small, while the systematic error is dominated by the uncertainty in the distance and is indicated in Figure 5 by arrows. The errors indicated in $T_{\text {eff }}$ are dominated by the uncertainty in the reddening. An overestimate of the reddening $E(B-V)$ by $\sim 0.04 \mathrm{mag}$, which seems unlikely, could reproduce most of the discrepancy shown in Figure 5 through the resulting underestimates of $T_{\text {eff }}$.

Another area of concern is the validity of the relationships we have adopted between color, $T_{\text {eff }}$, and $\log g$. As discussed earlier, we have carefully checked the consistency of the predicted colors from Houdashelt et al. (2000) with those from Kurucz (1992) computed using the ATLAS code, and we have also examined the comparison with the empirical color- $T_{\text {eff }}-[\mathrm{Fe} / \mathrm{H}]$ relations for dwarfs and for giants established by Alonso et al. (1996, 1999). For $V-K$, the MARCS and Kurucz predictions are in very close agreement, while the empirical fits to the angular diameter measurements using the infrared flux method carried out by Alonso et al. yield a $T_{\text {eff }}$ about $50 \mathrm{~K}$ cooler for a fixed $V-K$ color in the relevant range.

In addition, the theoretical tracks utilized thus far do not include enhancement of the $\alpha$-elements, which is common in metal poor globular cluster giants. However, the Oenhanced tracks of Bergbusch \& VandenBerg (1992) do not fit any better for the nominal metallicity of M71. This is not surprising as Bergbusch \& VandenBerg show that to first order the effects of enhancing $\mathrm{O}$ are equivalent to using a model with scaled solar abundances with an appropriately calculated higher global metallicity. This would make the predicted RGB cooler, making the discrepancy slightly worse. Their latest $\alpha$-enhanced models given in VandenBerg et al. (2000) retain this behavior.

We know from Paper II the correct $[\mathrm{Fe} / \mathrm{H}]$ for $\mathrm{M} 71$, and we will shortly know from (Ramírez \& Cohen 2001; hereafter Paper III) the $\alpha$-element enhancements. With that information, plus the stellar parameters of Table 3, once any small remaining discrepancies between the predicted and observed stellar parameters is understood, one can check for consistency with the new $\alpha$-enhanced tracks of VandenBerg et al. (2000).

The total effect under consideration (i.e., the discrepancy between the theoretical stellar isochrones and the behavior of the observed cluster sample in M71 shown in Fig. 3) is only $\sim 50-100 \mathrm{~K}$ in $T_{\text {eff }}$. There are several possible contributions on the observational side which may be large enough to explain it, including an error in the adopted reddening for the cluster and uncertainties in the relation utilized between color and stellar atmospheric parameters. Hence we have chosen to wait for more such comparisons to be carried out in the domain of $T_{\text {eff }}, \log g$ for additional clusters in future papers before speculating further on this issue.

\section{LOOKING FORWARD}

With this information in hand, we are ready to carry out an abundance analysis based on measurements of equivalent widths from the HIRES spectra of the M71 sample. An 
analysis of the $\mathrm{Fe}$ abundances for this sample of M71 stars is presented in the next paper in this series (Paper II).

The entire Keck/HIRES and LRIS user community owes a huge debt to Jerry Nelson, Gerry Smith, Steve Vogt, Bev Oke, and many other people who have worked to make the Keck Telescope and HIRES and LRIS a reality and to operate and maintain the Keck Observatory. We are grateful to the W. M. Keck Foundation for the vision to fund the construction of the W. M. Keck Observatory. We thank Peter Stetson for supplying his M71 photometry in easily accessible form and Peter Hauschildt for calculating a grid of $\mathrm{H} \alpha$ profiles for us. Partial support was provided to M. M. B. by a Theodore Dunham, Jr., grant for Research in Astronomy and by the National Science Foundation under grants AST-98 19614 to J. G. C. and AST-96 24680 to M. M. B.
Alonso, A., Arribas, S., \& Martinez-Roger, C. 1996, A\&A, 313, 873 1999, A\&AS, 140, 261

Arp, H. C., \& Hartwick, F. D. A. 1971, ApJ, 167, 499

Barklem, P. S., Piskunov, N., \& O'Mara, B. J. 2000, A\&A, 363, 1091

Bergbusch, P. A. \& VandenBerg, D. A. 1992, ApJS, 81, 163

Briley, M. M., \& Cohen, J. G. 2001, AJ, 122, 242

Briley, M. M., Smith, G. H., \& Claver, C. F. 2001, AJ, submitted

Briley, M. M., Smith, V. V., Suntzeff, N. B., Lambert, D. L., Bell, R. A., \& Hesser, J. E. 1996, Nature, 383, 604

Cannon, R. D., Croke, B. F. W., Bell, R. A., Hesser, J. E., \& Stathakis, R. A. 1998, MNRAS, 298, 601

Charbonnel, C. 1994, A\&A, 282, 811

. 1995, ApJ, 453, L41

Cohen, J. G. 1979, ApJ, 231, 751 1983, ApJ, 270, 654 1999, AJ, 117, 2434

Cohen, J. G., Frogel, J. A., Persson, S. E., \& Elias, J. H. 1981, ApJ, 249, 481

Cohen, J. G., \& Sleeper, E. C. 1995, AJ, 109, 242

Cook, K., Mateo, M., Olszewski, E. W., Vost, S. S., Stubbs, C., \& Diercks, A. 1999, PASP, 111, 306

Côté, P., Mateo, M., Olszewski, E. W., \& Cook, K. H. 1999, ApJ, 526, 147

Cudworth, K. M. 1985, AJ, 90, 65

Frogel, J. A., Persson, S. E., \& Cohen, J. G. 1979, ApJ, 227, 499

Geffert, M., \& Maintz, G. 2000, A\&AS, 144, 227

Gratton, R. G., et al. 2001, A\&A, 369, 87

Gustafsson, B., Bell, R. A., Eriksson, K., \& Nordlund, A. 1975, A\&A, 42, 407

Harris, W. E. 1996, AJ, 112, 1487

Hauschildt, P. H., Allard, F., Ferguson, J., Baron, E., \& Alexander, D. R. 1999, ApJ, 525, 871

Houdashelt, M. L., Bell, R. A., \& Sweigart, A. V. 2000, AJ, 119, 1448

King, J. R., Stephens, A., Boesgaard, A. M., \& Deliyannis, C. P. 1998, AJ, 115,666

Kraft, R. P. 1994, PASP, 106, 553

\section{REFERENCES}

Kurucz, R. L. 1992, CD-ROM 13, ATLAS9 Stellar Atmosphere Programs and $2 \mathrm{~km} / \mathrm{s}$ Grid (Cambridge: Smithsonian Astrophys. Obs.)

Landolt, A. R. 1992, AJ, 104, 340

Leep, E. M., Oke, J. B., \& Wallerstein, G. 1987, AJ, 93, 338

Mateo, M., Olszewski, E. W., Vogt, S. S., \& Keane, M. J. 1998, AJ, 116, 2315

McCarthy, J. K. 1988, Ph.D. thesis, Caltech

McClean, I. S., et al. 1998, SPIE, 3354, 566

2000, PASP, in press

Murphy, D. C., Persson, S. E., Pahre, M. A., Sivaramakrishnan, A., \& Djorgovski, S. G. 1995, PASP, 107, 1234

Oke, J. B., et al. 1995, PASP, 107, 375

Persson, S. E., Murphy, D. C., Krzeminsky, W., Roth, M., \& Rieke, M. J. 1998, AJ, 116, 2475

Peterson, R. C., \& Latham, D. W. 1986, ApJ, 305, 645

Pinsonneault, M. 1997, ARA\&A, 35, 557

Ramírez, S., \& Cohen, J. G. 2001, AJ, submitted

Ramírez, S., Cohen, J. G., Buss, R., \& Briley, M. 2001, AJ, 122, 1429

Schlegel, D. J., Finkbeiner, D. P., \& Davis, M. 1998, ApJ, 500, 525

Shortridge, K. 1993, FIGARO User's Manual (version 2.4; Chilton: Rutherford Appelton Lab.)

Sneden, C., Kraft, R. P., Langer, G. E., Prosser, C. F., \& Shetrone, M. D. 1994, AJ, 107, 1773

Sneden, C., Kraft, R. P., Prosser, C. F., \& Langer, G. E. 1991, AJ, 102, 2001

Stetson, P. B. 2000, PASP, 112, 925

Suntzeff, N. B., \& Smith, V. V. 1991, ApJ, 381, 160

Sweigart, A. V., \& Mengel, J. G. 1979, ApJ, 229, 624

Tonry, J. L., \& Davis, M. 1979, AJ, 84, 1511

VandenBerg, D. A., Swenson, F. J., Rogers, F. J., Iglesias, C. A., \& Alexander, D. R. 2000, ApJ, 532, 430

van’t Veer-Menneret, C., \& Megessier, C. 1996, A\&A, 309, 879

Vogt, S. E., et al. 1994, SPIE, 2198, 362

Yi, S., Demarque, P., Kim, Y.-C., Lee, Y.-W., Ree, C. Lejeune, Th., \& Barnes, S. 2001, ApJ, submitted (astro-ph/0104292) 